\title{
MÉXICO: ESTABILIDAD DE PRECIOS Y LIMITACIONES DEL CANAL DE CRÉDITO BANCARIO
}

\author{
Josefina León y César Alvarado*
}

Fecha de recepción: 24 de julio de 2014. Fecha de aceptación: 24 de noviembre de 2014.

\begin{abstract}
RESUMEN
En México la política monetaria ha sido eficaz para lograr la estabilidad de los precios; sin embargo, esto no se ha visto reflejado en crecimiento económico y generación de empleo. En este artículo se estudia el canal del crédito bancario, tema que ha generado mucho interés en la literatura económica; se analiza el mercado de crédito para el caso de México. La hipótesis que se plantea es que la existencia de un oligopolio bancario constituye un factor importante que limita la generación de crédito en la economía mexicana, lo que genera efectos negativos en la actividad económica.
\end{abstract}

Palabras clave: estabilidad de precios, instituciones financieras, sistema bancario, crédito bancario, política monetaria.

Clasificación JEL: E02, E42, E52, E58, G21.

\section{Mexico: Price Stability and the Limitations of the Bank Credit Channel}

\begin{abstract}
In Mexico, monetary policy has proved effective in achieving price stability, but this has not been reflected in economic growth and job creation. This article studies the bank credit channel, a topic that has stirred up much interest in economic literature, specifically analyzing the credit market in Mexico. It hypothesizes that the existence of a banking oligopoly is a key factor that limits the creation of credit in the Mexican economy, which negatively impacts economic activity.
\end{abstract}

Key Words: Price stability, financial institutions, banking system, bank credit, monetary policy.

\footnotetext{
* Universidad Autónoma Metropolitana, México. Correo electrónico: leon2josefita@hotmail.com y dalvaradog@hotmail.com, respectivamente.
} 
Josefina León y César Alvarado

\section{MEXIQUE: STABILITÉ DES PRIX ET LIMITATIONS DU CANAL DE CRÉDIT BANCAIRE \\ Résumé}

Au Mexique, la politique monétaire a été efficace pour parvenir à la stabilité des prix ; pourtant, ceci ne s'est pas reflété dans la croissance économique ni la création d'emplois. Dans cet article, on étudie le canal du crédit bancaire, sujet qui a suscité grand d'intérêt dans la littérature ès économie ; le marché du crédit est analysé pour le cas du Mexique. L'hypothèse qui est formulée est que l'existence d'un oligopole bancaire constitue un facteur important qui limite la génération de crédit dans l'économie mexicaine, ce qui a des effets négatifs sur l'activité économique.

Mots clés: stabilité des prix, institutions financières, système bancaire, crédit bancaire, politique monétaire.

\section{MÉXICO: ESTABILIDADE DE PREÇOS E LIMITAÇÓES DO CANAL DE CRÉDITO BANCÁRIO \\ Resumo}

No México, a política monetária foi eficaz para alcançar a estabilidade dos preços; porém, isso não se viu refletido em crescimento econômico e geração de emprego. Neste artigo se estuda o canal de crédito bancário, tema que tem gerado muito interesse na literatura econômica, e se analisa o mercado de crédito para o caso do México. A hipótese que se planteia é que a existência de um oligopólio bancário se converte num fator importante que limita a geração de crédito na economia mexicana, o que gera efeitos negativos na atividade econômica.

Palavras-chave: estabilidade de preços, instituições financeiras, sistema bancário, crédito bancário, política monetária.

\footnotetext{
墨西哥的物价稳定与对银行信贷渠道的限制

摘要:

墨西哥的货币政策在稳定物价方面是有效的，但是在实现经济增长、扩大就业方面 的功能未能充分发挥出来。本文研究了墨西哥银行信贷渠道问题, 这个话题在经济 研究文献中激起了很大的兴趣, 特别是在分析墨西哥的信贷市场方面。本文假设银 行业属于垄断行业, 这个关键因素限制了墨西哥经济中的信贷创造, 结果是对经济 活动造成了负面影响。

关键词：价格稳定金融机构银行体系银行信贷货币政策
} 


\section{INTRODUCCIÓN}

En materia de política económica, la crisis de balanza de pagos que México sufrió en 1994-1995 implicó un cambio en el régimen cambiario; el gobierno se vio obligado a transitar de un tipo de cambio fijo con bandas de flotación a un tipo de cambio flexible, en el que ha predominado, como en la mayoría de los países que aplican este tipo de regímenes, la flotación intervenida o "sucia". A raíz de esta modificación, la política monetaria se convierte en pieza fundamental de la estabilidad macroeconómica. ${ }^{1}$ Posteriormente, con la crisis financiera mundial de 2008-2009 se pone en evidencia que para lograr el objetivo de estabilidad de precios, el Banco de México (Banxico) utiliza como instrumentos de la política monetaria no solo la tasa de interés de fondeo bancario, ${ }^{2}$ sino también el tipo de cambio y las operaciones de mercado abierto.

El esquema actual de metas de inflación que Banxico aplica desde principios del siglo XXI se inscribe en el marco del nuevo paradigma monetario ${ }^{3}$ desarrollado por los autores de la nueva síntesis neoclásica. El modelo de inflación objetivo se establece en 2001 y en 2002 se señala el criterio en relación con el rango de la tasa de variación de los precios. Se espera que las expectativas de inflación de los agentes económicos $\hat{P}^{e}$ coincidan con el objetivo establecido por Banxico. El criterio en relación con el rango establecido se debe a que desea tener una inflación similar a la de los países socios del Tratado de Libre Comercio de América del Norte (TLCAN), Estados Unidos y Canadá.

$$
\hat{P}^{e}=\hat{P}=3 \pm 1 \%
$$

La política monetaria ha sido eficaz para controlar el crecimiento de los precios y, en términos relativos, se ha logrado un proceso de convergencia hacia las tasas de inflación de Estados Unidos y Canadá. De acuerdo con el

1 Como señala Bernanke (2014), expresidente de la Reserva Federal (Fed) de Estados Unidos, los bancos centrales tienen fundamentalmente dos objetivos: lograr la estabilidad macroeconómica y mantener la estabilidad financiera; la estabilidad macroeconómica implica conseguir un crecimiento estable de la economía, evitando grandes oscilaciones, y mantener la inflación baja y estable. Esa es la función económica de un banco central y su principal herramienta es la política monetaria. En lo que tiene que ver con la estabilidad financiera la herramienta principal de los bancos centrales para combatir los pánicos bancarios o las crisis financieras es la provisión de liquidez, es decir, actuar como prestamista de última instancia.

2 A partir del 21 de enero de 2008 se adopta como objetivo operacional a la tasa de interés interbancaria a un día ("tasa de fondeo bancario").

3 Para mayor información sobre este tema, véase Perrotini (2007). 
banco central, primero debe lograrse la estabilidad de precios y después se espera que la economía retome el sendero del crecimiento económico, ideas inspiradas en autores como Milton Friedman, principal representante de la escuela monetarista. ${ }^{4}$ Desafortunadamente, en nuestro país la estabilidad de precios no se ha acompañado de crecimiento económico y generación de empleo. De la crisis de 1995 a la actualidad ya han pasado casi dos décadas y nos encontramos todavía en lo que para los monetaristas sería la etapa de corto plazo, es decir, soportando un costo social que ha sumido al país en una situación de estancamiento económico con estabilidad de precios.

¿Por qué la política monetaria no ha incidido de manera favorable en el crecimiento económico y la generación de empleo? Esto tiene que ver, básicamente, con el mecanismo de transmisión de la política monetaria, que considera las relaciones que se establecen entre los cambios de la política monetaria y su efecto en la producción, el empleo y los precios. "El mecanismo de transmisión de la política monetaria se puede dividir en dos elementos: el impacto de los cambios monetarios en la demanda agregada, y el efecto de las variaciones en la demanda agregada sobre la producción, el empleo y los precios" (Bain y Howells, 2003: 171).

El análisis del mecanismo de transmisión incluye diferentes canales y en la bibliografía macroeconómica ortodoxa normalmente se abordan cuatro: el canal de la tasa de interés, el canal del tipo de cambio, el canal de precios de otros activos y el canal de crédito. ${ }^{5}$ Los impactos de la política monetaria tienen efectos tanto en el mercado interno como en el sector externo, ya en otro trabajo hemos abordado esta temática. ${ }^{6} \mathrm{El}$ propósito de este artículo es explicar de manera sencilla el canal de crédito bancario, para lo cual nos basamos en el modelo básico de Bernanke y Blinder (1988), luego analizamos el mercado del crédito para el caso de México. La hipótesis que planteamos aquí es que la existencia de un oligopolio bancario, que se refleja, por ejemplo, en el alto

4 Véase, por ejemplo, los artículos de M. Friedman de 1968 y 1976, en los que analiza el rol de la política monetaria en el sistema económico y distingue los impactos de las variaciones monetarias a corto y largo plazo. De su planteamiento teórico se deduce que para controlar la inflación debe aplicarse una política monetaria contractiva que en el corto plazo inevitablemente ocasionará un costo social en términos de pérdida de empleo y caída en el crecimiento económico, pero que en el largo plazo, una vez que los agentes económicos ajusten sus expectativas de precio hacia la baja, el crecimiento económico y el empleo se recuperarán a sus niveles asociados con la tasa natural de desempleo.

5 Para una descripción detallada de cada uno de estos canales véase Mishkin (1995).

6 Véase León (2012). 
grado de concentración en la captación de depósitos que constituye un factor importante que limita la generación de crédito en la economía mexicana, lo que genera efectos negativos sobre todo para las micro, pequeñas y medianas empresas, que son las que más dependen del crédito bancario.

El canal de crédito se refiere a cómo afecta la política monetaria a los préstamos bancarios y, a la vez, los préstamos bancarios al ingreso mediante el crédito que las instituciones bancarias otorgan a las empresas y familias. De acuerdo con Bernanke y Gertler (1995) existen varios modelos que formalizan el canal de crédito, pero a grandes rasgos pueden dividirse en dos tipos: el canal de los préstamos bancarios (the bank lending channel) y el canal de los balances (the balance sheet channel); el primero es el que se expondrá en este trabajo.

El canal de los balances se refiere a cuando las empresas deben contar con garantías para que los bancos les presten. El valor de estas garantías cambia a lo largo del ciclo económico, lo que a su vez afecta la capacidad de endeudamiento de las empresas. Los movimientos en la tasa de interés modifican el valor de los activos y pasivos de los deudores actuales y potenciales, afectando su situación patrimonial y también su solvencia. Una política monetaria contractiva puede aumentar el gasto en intereses de una empresa, reduciendo sus flujos netos de caja y empeorando su situación financiera. Al mismo tiempo, el aumento en las tasas de interés se asocia normalmente con una caída en el precio de los activos, disminuyendo el valor del colateral que pueden presentar los deudores. De esta forma, la posición financiera de las empresas empeora y se les vuelve más difícil conseguir financiamiento, lo que reduce la inversión. ${ }^{7}$

El trabajo está integrado por tres apartados. En el primero se plantean brevemente las ideas de algunos autores representativos que han hecho énfasis en la importancia del crédito bancario en el sistema económico; para explicar el funcionamiento del canal de crédito se presenta el modelo básico de Bernanke y Blinder (1988). En el segundo se analiza el sistema bancario mexicano y se muestra que constituye una estructura de mercado de competencia imperfecta; aquí se analizan dos variables: el grado de concentración en la captación de depósitos bancarios y los diferenciales existentes entre las tasas de interés activas en relación con las tasas pasivas. Finalmente, se presentan las conclusiones del estudio. 


\section{EL CANAL DEL CRÉDITO BANCARIO}

\subsection{Antecedentes históricos de la importancia del crédito bancario}

Los préstamos son importantes en los mercados financieros porque muchas empresas y familias se financian con créditos bancarios; la banca es fuente de financiamiento de la inversión productiva, la adquisición de vivienda y bienes de consumo duradero. Por lo tanto, la generación o contracción del crédito bancario incide en la generación de ciclos económicos; el crédito es una variable de carácter procíclico, es decir, en los periodos de expansión económica normalmente se incrementa el crédito y en las fases de contracción económica, puede llevar a la crisis o depresión económica, el crédito bancario se reduce porque la tasa de riesgo aumenta. La importancia del crédito bancario puede encontrarse en diferentes autores y enfoques teóricos.

Para I. Fisher (1867-1947) las fluctuaciones del dinero circulante y la disponibilidad de crédito, además de causar inflaciones y deflaciones, explica los altibajos de la actividad económica y el empleo. Cada vez estaba más convencido de que una mejor gestión de la moneda permitiría "suavizar las fluctuaciones cíclicas" ${ }^{8}$ Por ejemplo, en la fase descendente del ciclo económico señala: "cuando los precios están cayendo es porque antes se han reducido los gastos, lo cual disminuye los beneficios. En consecuencia, durante periodos de deflación, caen los beneficios y se incrementan las quiebras, lo que aumenta la tasa de desempleo" (Fisher, 1973: 498). En su perspectiva una medida para prevenir de manera sustancial el desempleo era mantener estable el poder adquisitivo de la moneda, es decir, mantener la estabilidad en el nivel general de los precios.

En su artículo denominado: "A Statistical Relaltion Between Unemployment and Price Change", publicado en 1926 y que posteriormente fue reeditado por Journal of Political Economy, en 1973, Fisher muestra que hay una relación causal entre inflación y empleo o deflación y desempleo: que las subidas y bajadas de empleo son los efectos, en gran medida, de las subidas y caídas de precios, debido a su vez a la inflación y la deflación generadas por las variaciones en el dinero y el crédito. ${ }^{9}$ (2014: 332).

9 Véase Fisher (1973: 502). 
Fisher publicó en 1928 The Money Illusion, obra fundamental para el entendimiento de los orígenes y consecuencias de lo que él llamó la ilusión de una moneda estable en un sistema patrón oro. El autor muestra que el supuesto de valor estable de las monedas no era más que una mera ilusión, ya que en el momento en que las naciones fijaban sus monedas oficiales a un peso específico respecto al oro y garantizaban su convertibilidad, no les permitía mantener su poder adquisitivo con respecto a las mercancías cuando el nivel de precios cambiaba, y como el comercio se solía realizar básicamente con este medio de intercambio monetario entonces las transacciones no tenían necesariamente punto referencial con sus valores, lo que generaba desequilibrios en la balanza comercial. Para entender este problema, Fisher plantea los números índices ${ }^{10}$ como un mecanismo para medir el valor real de las monedas, estableciendo algunas referencias históricas, fundamentalmente el caso de Alemania durante y después de la Primera Guerra Mundial.

Fisher insistía en que la estabilidad de la moneda era un asunto básicamente social, ya que: "Si queremos evitar que nuestra basta superestructura de crédito se desmorone periódicamente sobre nuestras cabezas, debemos ver la actividad bancaria como algo más que un negocio privado: es un importante servicio público" (Nasar, 2014: 334).

R.G. Hawtrey (1879-1975) es el exponente más conocido de la teoría monetaria del ciclo, en su perspectiva la elasticidad del crédito bancario es la causa de los ciclos económicos. En el planteamiento de este autor no existe la figura del banco central, el sistema bancario está integrado por bancos comerciales del sector privado, el análisis se realiza bajo el sistema monetario del patrón oro. ${ }^{11}$ En este contexto, la fase de auge de un ciclo económico inicia cuando los comerciantes empiezan a observar que el nivel de sus inventarios empieza a bajar a niveles no deseados; por lo tanto, los empiezan a aumentar haciendo pedidos nuevos a los productores. Pero en lugar de financiarlos con un aumento del ahorro, deciden pedir un crédito a los bancos, lo que les genera una deuda; por su parte los empresarios van a pagar a los proveedores también con crédito bancario a través de expedir un cheque, de modo tal que las existencias de los comerciantes aumentan sin necesidad de que disminuya el nivel de consumo corriente.

10 Para Fisher los números índices eran el mejor mecanismo para medir el poder adquisitivo de las monedas, ya que proporcionan en cifras porcentuales la variación de precios que había sufrido en promedio un cierto número de mercancías-tipo durante un periodo de tiempo; la inestabilidad del valor de las monedas fue fundamental para la hipótesis de Fisher respecto a la inestabilidad del patrón oro.

11 Las ideas que planteamos aquí sobre Hawtrey se basan en un estudio anterior, véase León (2010). 
El aumento en el crédito bancario reduce el nivel de la tasa de interés monetaria, lo que permite a los negociantes incrementar las existencias de mercancías, esto genera un aumento en la demanda efectiva dando origen a la fase expansiva del ciclo económico, se demanda más trabajo y capital con el fin de incrementar la producción, que a su vez incrementa la actividad comercial y motiva a la demanda de nuevos créditos bancarios que otra vez se reflejarán en un ascenso en la demanda efectiva.

Esta fase se mantiene mientras se siga expandiendo el crédito, sin embargo, los bancos no pueden otorgar crédito en forma ilimitada debido a que se empieza a generar escasez en sus reservas, lo que los lleva a reducir el crédito. En el momento en el que el sistema económico enfrenta una restricción de crédito, la demanda efectiva se empieza a contraer, con la consecuente caída en los niveles de producción, empleo y precios. Hawtrey señala que si la restricción del crédito no ocurriera, la fase de auge del ciclo podría prolongarse indefinidamente, a expensas de un aumento indefinido de los precios y del abandono del patrón oro.

Por lo anterior, considera que lo más adecuado es adoptar políticas que contribuyan a estabilizar los precios, creemos que en el autor hay un argumento implícito sobre la necesidad de contar con un banco central cuando señala que las autoridades responsables controlen el crédito y estén dispuestas a cooperar (Hawtrey, 1928). "El banco central debe de estar encargado de controlar la emisión monetaria y la tasa de interés es el factor más importante para estabilizar el valor de la moneda o de manera equivalente, controlar el crecimiento de los precios" (León, 2010: 111).

El ciclo del crédito fue una preocupación central de J.M. Keynes en el Tratado del dinero (1930), obra en la que la influencia de K. Wicksell es reconocida explícitamente por el autor al referirse a los conceptos de: ahorro, inversión, tasa natural de interés y tasa de interés del mercado. En la Teoría General (1984: 10) Keynes señala que las ideas planteadas en su libro de 1930 corresponden a la teoría económica clásica convencional, pues se considera dado el nivel de producción; Keynes plantea que las fluctuaciones en el producto y el empleo son el resultado de insuficiencias en la demanda efectiva, provocadas básicamente por la inestabilidad en la inversión, cuyos movimientos están estrechamente relacionados a los mercados especulativos. Pero como señala Levy (2011), Keynes en la Teoría General omite la demanda de dinero por el motivo de financiamiento para resaltar la importancia del motivo de especulación en la inestabilidad de la demanda de dinero, responsable de las variaciones de la tasa de interés y el ingreso. El motivo de financiamiento se reintroduce en otros trabajos (1937a, 1937b y 1939). 
Con base en los artículos de 1937, Levy señala que para Keynes la provisión de financiamiento proviene de dos fuentes: los créditos bancarios y las "nuevas emisiones", las cuales son indistintas. En el caso de la provisión de liquidez por parte de la banca "el proceso inicia con la decisión de gastar (inversión ex ante), la cual requiere de la liquidez que pueden otorgar los bancos, con base en un fondo "revolvente" (propuesta de Robertson), definido como un monto de liquidez relativamente constante "donde un empresario puede agotar los recursos del fondo y otro reaprovisionarlos, liquidando sus deudas, después de haber realizado la inversión" (Keynes, 1937a: 246). Es decir, dado un monto de liquidez, se puede generar un proceso continuo de financiamiento si las deudas adquiridas para financiar la producción son saldadas. Empero, si se rompe la igualdad entre inversión ex ante y ex post debe inyectarse mayor liquidez al fondo "revolvente" (adelanto de efectivo de una deuda). Keynes supuso que los bancos pueden satisfacer dicha demanda otorgando créditos, independientes de recursos reales, aunque alerta que los banqueros también pueden aumentar las tasas de interés, generándose una congestión en el mercado bancario" (Levy, 2011: 114-115).

La escuela poskeynesiana constituye una corriente heterodoxa y se integra por un grupo heterogéneo de economistas; ${ }^{12}$ B. Snowdon y H. Vane (2005: 452) señalan que, de acuerdo con Holt (1997), la mayoría de los economistas que se hacen llamar poskeynesianos se dividen tradicionalmente en dos amplios grupos: i) La corriente Europea o de Cambridge, Reino Unido; incluye los trabajos asociados con economistas como Geoff Harcourt, Richard Kahn, Nicholas Kaldor, Michael Kalecki, Joan Robinson y Piero Sraffa. Este grupo ha enfatizado el comportamiento y funcionamiento de la economía real, mientras que ignora, o al menos minimiza, las implicaciones monetarias y financieras. ii) La corriente americana; incluye autores como Victoria Chick, Alfred Eichner, Jan Kregel, Hyman Minsky, Basil Moore, George Shackle, Sydney Weintraub y Paul Davidson. ${ }^{13}$ Algunos, pero no todos los integrantes

Marc Lavoie (2004) señala que los poskeynesianos modernos se inspiran principalmente en los trabajos y métodos desplegados por los economistas que se codeaban con Keynes en la época en que se encontraba escribiendo su Teoría General (1936), como Roy Harrod o Joan Robinson, o que han contribuido a lo que se llamó la "Escuela de Cambridge" en los años cincuenta y sesenta, como Nicholas Kaldor, Michael Kalecki y Piero Sraffa. Los poskeynesianos, como los regulacionistas, también están estrechamente ligados con los institucionalistas, en especial con los que se han inspirado en las ideas de Thorstein Veblen o J. K. Galbraith. Se distinguen tres corrientes principales: fundamentalistas, sraffianos y kaleckianos.

13 Si bien es cierto que Holt etiqueta a este grupo como la corriente "americana", esta clasificación la hace a partir del estilo y énfasis en el análisis económico, y no en la nacionalidad. Así por ejemplo, 
de este grupo, han concentrado su atención en el impacto de la incertidumbre y las influencias monetarias y financieras en la economía.

Las teorías monetarias poskeynesianas, de acuerdo con Pierre Piégay y Philippe Rochon (2006), se clasifican en tres tipos: 1) teoría monetaria poskeynesiana, 2) teoría del circuito monetario y 3) la escuela schmittiana del circuito. En estas teorías se liga la creación monetaria a la producción y el crédito de los bancos comerciales desempeña un papel crucial en el sistema económico.

En términos muy generales la teoría monetaria poskeynesiana señala que en una economía monetaria hay una interrelación importante entre el dinero y la actividad económica, la oferta monetaria es endógena, el dinero es no neutral tanto en el corto como en el largo plazo; y la tasa de interés monetaria de corto plazo, más que representar el costo del dinero como en la teoría neoclásica, constituye una variable distributiva. Asimismo, la generación de ingreso no implica necesariamente un gasto equivalente, dada la teoría de la preferencia por liquidez y la existencia de incertidumbre en los diferentes mercados.

Por su parte, en el marco de la nueva economía keynesiana autores como Ben Bernanke y Mark Gertler (1995) señalan que la influencia de la política monetaria sobre la economía real va más allá del canal tradicional de la tasa de interés, que opera a través de la demanda agregada y afecta las decisiones de consumo e inversión. Ellos hacen énfasis en el canal de crédito, mecanismo que como veremos en el siguiente apartado, amplifica y propaga los efectos del canal tradicional de la política monetaria. El artículo de Bernanke (1983) es complementario al trabajo de M. Friedman y A. Schwartz (1963), quienes hicieron hincapié en el impacto monetario de las quiebras bancarias en la década de los treinta; ${ }^{14}$ en este caso el autor se centra en los aspectos no monetarios del sector financiero asociados con el crédito y su impacto en la producción, considerando los problemas de los deudores y del sistema bancario. El autor demuestra que las perturbaciones financieras de 1930-1933 disminuyeron la eficiencia del proceso de asignación de créditos, originando un mayor costo y una menor disponibilidad de crédito, lo que contribuyó en

George Shackle es británico y Victoria Chick, aunque nacida en Estados Unidos, ha realizado su carrera profesional en Inglaterra (véase Snowdon y Vane, 2005: 452).

14 De acuerdo con Bernanke $(2014: 38,39)$ la Fed no cumplió su mandato de prestamista de última instancia y ante los pánicos bancarios respondió de manera inadecuada, gran parte de los bancos quebraron (casi 10 mil) en la década de los treinta. En 1933 llega al poder Franklin D. Roosevelt y abandona el patrón oro, posteriormente en 1934 crea el fondo de garantía de depósitos (FDIC), lo que evitó las quiebras bancarias; las medidas anteriores hicieron que la política monetaria fuera menos rígida, lo que produjo un repunte en la economía en 1933 y 1934. 
el descenso de la demanda agregada y puede explicar la duración y profundidad de la Gran Depresión.

De acuerdo con lo que plantean autores como Karl Brunner y Allan Meltzer (1993), la intermediación y los mercados de crédito cumplen un papel en la transmisión de la política monetaria y fiscal hacia los mercados de activos y productos. Sin embargo, en una gran cantidad de modelos macroeconómicos no se considera el mercado de crédito, lo cual constituye un factor que limita el alcance de sus conclusiones. Por ejemplo, en el modelo Is-LM se asume que existen dos activos: dinero y bonos, de este modo, el único tipo de interés que aparece es el de los bonos y éste constituye el único canal de transmisión de la política monetaria. Pero este canal es insuficiente para explicar los efectos que las variaciones monetarias generan en las variables reales y nominales, en un mundo en donde hay imperfecciones tanto en los mercados financieros como en el funcionamiento de las empresas.

Hay muchas formas en que las empresas pueden financiar sus inversiones: en el mercado de capitales, emitiendo bonos o acciones; ir directamente a un banco a pedir un crédito ${ }^{15} \mathrm{o}$, finalmente, a través del endeudamiento externo. Un aspecto importante para el análisis del canal de crédito es que el crédito bancario y el resto de instrumentos de deuda no son sustitutos perfectos; además, no todas las empresas tienen acceso a las diferentes formas de financiamiento, las micro, pequeñas y medianas empresas normalmente van a estar limitadas al crédito bancario o al crédito informal en las cajas de ahorro popular. Por lo anterior, el canal de crédito por parte de los bancos hace que este tipo de empresas vean un límite a su financiamiento. Por el contrario, en el caso de las empresas grandes no tienen problemas para ingresas al mercado de capitales, dada su solvencia, su rentabilidad y su historia.

El canal del crédito funciona de la siguiente manera: una política monetaria expansiva incrementa los depósitos bancarios y con esto asciende la cantidad disponible para los préstamos bancarios, lo que genera un aumento en la inversión, el consumo de bienes duraderos y el producto agregado. La cadena de eventos se describe a continuación.

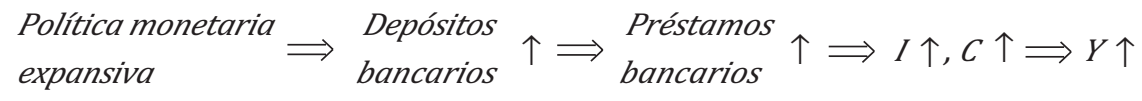
cado financiero. El mercado de capitales es donde se comercian instrumentos de renta fija y variable a más de un año, mientras los bancos, que igual participan en el mercado de capitales, se especializan en ofrecer créditos y captar depósitos. El mercado a menos de un año es el mercado monetario. Véase De Gregorio (2007: 688). 
En la década de los setenta las ideas del monetarismo señalaban que la política monetaria desempeña un papel relevante en la actividad económica, pero no son evidentes las vías por medio de las cuales afecta al producto en el corto plazo; este aspecto es importante debido a la presencia de estudios que sostienen que la demanda agregada es inelástica ante variaciones de la tasa de interés, sin embargo, se planteaba que dicha demanda sí es muy sensible ante alteraciones en el consumo y la inversión. Una alternativa fue analizar los efectos positivos que el dinero tiene sobre la actividad económica y los impactos que pudieran perdurar en el tiempo.

De Gregorio (2007) presenta un estudio para distintos países sobre la correlación existente entre el crédito bancario como porcentaje del PIB y el PIB per cápita, el estudio toma los datos disponibles del Fondo Monetario Internacional (FMI) durante el periodo de 1990 a 2004, considerando únicamente a países cuya población es mayor a un millón de habitantes y cuyo ingreso per cápita rebase mil dólares anuales. El trabajo demuestra que los bancos siempre elevan el crédito en los periodos de auge de la actividad económica y lo disminuyen en las recesiones, además encuentra una correlación positiva entre las variables estudiadas cuya media es de 0.5 para el conjunto de países analizados.

\subsection{EL MODELO DE BERNANKE Y BLINDER}

El modelo Is-LM plantea el papel de la política monetaria mediante el canal de la tasa de interés, "pero carece de mercados para créditos o préstamos, con lo que los argumentos sobre la importancia del racionamiento del crédito o los préstamos bancarios no pueden ser evaluados a la luz del marco Is-LM" (Brunner y Meltzer, 1995: 88).

Bernanke y Blinder (1988) desarrollan una variante del modelo Is-LM, en la que se incluyen tres activos: dinero, crédito y bonos. El modelo supone que los agentes económicos no usan dinero circulante, por tanto, la demanda de dinero está constituida sólo por depósitos, los bancos mantienen una reserva por un monto $\tau$ de estos depósitos, dicho monto se asume constante, y se supone que los depósitos no pagan intereses; se trata de un modelo con precios fijos iguales a la unidad, de tal suerte que las cantidades reales y nominales son igual. Finalmente se afirma que el mercado de dinero se describe por la función LM. "Un banco central puede establecer sus objetivos intermedios tanto en términos de un agregado monetario como crediticio. La bondad de la elección depende de la estabilidad relativa de las demandas de dinero y 
crédito. Si las perturbaciones que afectan a la demanda de dinero son empíricamente más relevantes que las perturbaciones que afectan a la demanda de crédito, entonces una política basada en un objetivo intermedio formulado en términos de un agregado crediticio tiene más fundamento que una formulada en términos de un agregado monetario y viceversa" (Vega, 1992: 19).

Se asume que tanto prestamistas como prestatarios eligen entre bonos y préstamos con base en el rendimiento que cada uno de ellos genera. Sea $\rho$ la tasa de interés sobre los préstamos y sea $i$ la tasa de interés de los bonos. Por tanto la demanda de préstamos se define de la siguiente manera:

$L^{d}=L(\rho, i, y) ; L_{y}>0, L_{\rho}<0, L_{i}>0$,

Donde $y$ denota el nivel de ingreso, por lo tanto, la demanda de crédito es en función de la tasa de interés de los bonos, del nivel de ingreso y de la tasa de interés del crédito. La ecuación (1) indica que un mayor nivel de ingreso genera un aumento en la demanda de préstamos, mientras que un incremento en la tasa de interés del crédito desincentiva la demanda de éste, y finalmente un incremento en la tasa de interés de los bonos produce un aumento en la demanda de crédito.

Por otro lado, para entender la oferta de préstamos se considera una versión simple de la hoja de balance bancaria.

\begin{tabular}{c|c}
\multicolumn{2}{c}{ Cuadro l } \\
\hline Activos & Pasivos \\
\hline Reservas (R) & Depósitos (D) \\
Bonos (B $\left.{ }^{b}\right)$ & \\
Préstamos $\left(L^{5}\right)$ &
\end{tabular}

Dado que las reservas se definen como la suma de los requerimientos de reserva, $(\tau D)$ y el exceso de reserva $(E)$, es decir $R=\tau D+E$, entonces la identidad contable se puede escribir como:

$$
B^{b}+L^{s}+E=D(1-\tau)
$$


La ecuación (2) afirma que la suma de bonos, crédito y exceso de reservas es igual a la cantidad de depósitos netos. El modelo supone que la composición de las proporciones de los activos dependerá de su tasa de retorno. La suma de estas proporciones se expresa como:

$$
\frac{B^{b}}{D(1-\tau)}+\frac{L^{s}}{D(1-\tau)}+\frac{E}{D(1-\tau)}=1
$$

Cada una de estas proporciones se define como una función de las tasas de interés.

$$
\beta(\rho, i)=\frac{B^{b}}{D(1-\tau)} ; \lambda(\rho, i)=\frac{L^{s}}{D(1-\tau)} ; \varepsilon(i)=\frac{E}{D(1-\tau)}
$$

Donde $\beta$ es función decreciente de $\rho$ y función creciente de $i$; en donde $\lambda$ es función creciente de $\rho$ y función decreciente de $i$. Finalmente el modelo asume, por simplicidad, que sólo $i$ influye en la demanda de exceso de reservas, donde $\varepsilon$ es una función decreciente de la tasa de interés de los bonos. Por tanto, la oferta de crédito queda determinada de la siguiente manera:

$$
L^{s}=\lambda(\rho, i) D(1-\tau)
$$

De tal suerte que el equilibrio en el mercado de crédito se puede expresar por medio de la siguiente ecuación:

$$
L(\rho, i, y)=\lambda(\rho, i) D(1-\tau)
$$

Sabemos por la argumentación anterior, que la cantidad total de reservas es igual a la suma de los requerimientos más el exceso de éstas, y que el exceso de reservas, a su vez se expresa como $\varepsilon(i) D(1-\tau)$. Por tanto, las reservas pueden ser definidas como:

$$
R=D[\varepsilon(i)(1-\tau)+\tau]
$$

Donde la demanda por depósitos $(D)$ es función creciente del ingreso y función decreciente de la tasa de interés de los bonos, es decir $D=D(i, y)$. Se denota como $m(i)$ a la expresión $[\varepsilon(i)(1-\tau)+\tau]^{-1}$. 
De esta manera la demanda de depósitos se puede expresar de la siguiente forma:

$$
D(i, y)=m(i) R
$$

El mercado restante es el mercado de bienes, el cual relaciona a las tasas de interés con la producción y se puede sintetiza con la siguiente expresión:

$$
y=Y(i, \rho) ; y_{i}<0 ; y_{p}>0
$$

Así, el modelo se compone de cuatro mercados: crédito, bonos, depósitos y bienes. Los autores del modelo utilizan el razonamiento hecho hasta ahora para derivar una curva que muestre no sólo el equilibrio en el mercado de bienes, sino también el equilibrio en el mercado de crédito. Para lograr esto, consideran los equilibrios en los mercados de crédito, depósitos y bienes, ecuaciones (6) (8) y (9), respectivamente y asumen que por la ley de Walras el mercado de bonos se encontrará en equilibrio.

Sustituyendo (8) en (6) y despejando para $\rho$ tenemos:

$$
\rho=\phi(i, y, R) ; \rho_{i}>0 ; \rho_{y}>0 ; \rho_{R}<0
$$

Sustituyendo (10) en (9) tenemos la siguiente expresión:

$$
y=\varphi(i, R)
$$

La ecuación (11), se denota como curva Cc (commodities and credit), es equivalente a la curva Is; sin embargo, bajo los argumentos utilizados en el modelo, el nivel de producción no sólo depende de la tasa de interés de los bonos, sino que ahora también es función del nivel de reservas, de esta manera todos los puntos sobre la curva CC representan combinaciones de producto y tasa de interés de los bonos que no sólo equilibran al mercado de bienes sino también al mercado de crédito. Como se afirmó al inicio, la curva LM no sufre ninguna modificación y se deriva de manera tradicional.

La gráfica 1, muestra la interacción de las curvas CC y LM cuando el banco central lleva a cabo una expansión monetaria por medio de operaciones de mercado abierto, esto produce un aumento de $R$ y a su vez un movimiento tanto de la curva $\mathrm{LM}$, de $L M_{0}$ a $L M_{1}$, como de la curva CC, de $C C_{0}$ hacia $C C_{1}$. Bajo el esquema tradicional del modelo IS-LM una política monetaria expansiva conduciría indudablemente a una caída en la tasa de interés de los bonos 


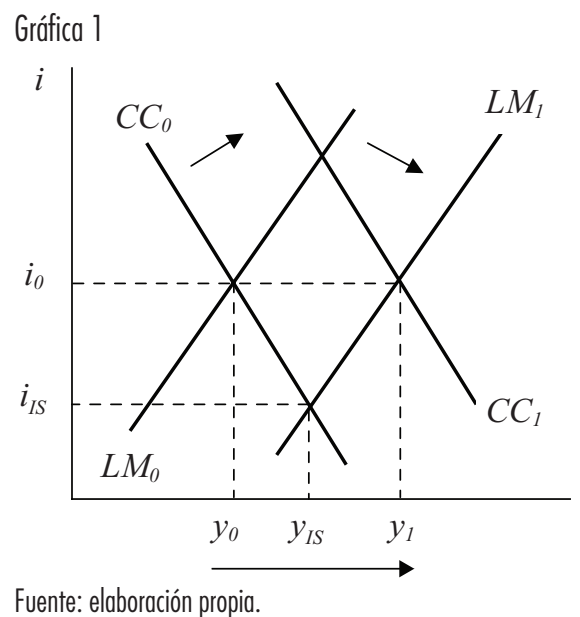

hasta un nivel igual a $i_{I S}$, mientas que el producto sólo lograría incrementarse hasta $y_{I S}$; sin embargo, como podemos apreciar en la gráfica 1 el efecto de una expansión monetaria sobre el producto es mayor cuando se considera al mercado de crédito, generando una expansión del producto hasta un nivel igual a $y_{1}$. Es necesario seńalar que en el esquema CC-LM, una expansión monetaria tienen efectos inciertos sobre la tasa de interés, debido a que el valor de ésta dependerá de la magnitud de los desplazamientos de las dos curvas y de sus respectivas pendientes. ${ }^{16}$

Por tanto, la conclusión es que un mercado de crédito eficiente potencializa los efectos de la política monetaria sobre el crecimiento económico.

\section{SISTEMA BANCARIO EN MÉXICO}

En esta sección se presentan algunos indicadores que dan cuenta del grado de concentración que existe en el sistema bancario mexicano, en particular nos enfocamos en la captación de depósitos y en el otorgamiento de crédito al sector privado. Asimismo se muestra la preferencia de los bancos comerciales en el otorgamiento de crédito a distintos sectores.

Con base en los datos de la Comisión Nacional Bancaria y de Valores (CNBV), podemos afirmar que en promedio, durante el periodo de 2001-2013,

16 En la gráfica 1 hemos mostrado, por simplicidad, el caso en que el nivel final de la tasa de interés de los bonos es igual al nivel inicial. 
alrededor del $80 \%$ de los bancos comerciales captaron el $12.57 \%$ de los depósitos, o en otras palabras, que el $20 \%$ de los bancos han captado el $87.43 \%$ de los depósitos. ${ }^{17}$ En 2001 sólo Bancomer y Banamex concentraban el 51.13\% de los depósitos: 28.98 y $23.17 \%$ respectivamente. Mientras que para febrero de 2014 el 51.48\% de los depósitos estuvieron en manos de Bancomer (22.26), Banamex (15.68) y Banorte (13.54).

En lo referente al otorgamiento de crédito, podemos decir que en promedio durante el mismo periodo el $18.13 \%$ de los créditos fueron otorgados por el $80 \%$ de los bancos, o bien que sólo $20 \%$ de los bancos otorgan el $81.87 \%$ de los créditos. En 2001 el $55.42 \%$ de crédito había sido concedido por tres bancos, con la siguiente distribución: Banamex, 25.85; Bancomer, 21.19 y Banorte, 8.38\%.

Un coeficiente común para medir el grado de concentración es el coeficiente de Gini, el cual es un número que oscila entre cero y uno; un valor igual a cero indica una situación de competencia perfecta y un valor igual a uno señala una situación de monopolio. En la gráfica 2 podemos observar la evolución del índice del sistema bancario mexicano; en ella se muestran tanto la concentración de depósitos como la concentración del otorgamiento de crédito. Un aspecto importante es que de acuerdo al padrón de banca múltiple registrado por la CNBV, en 2001 había 30 bancos y, para 2014, como señalamos con anterioridad, había 43 bancos, con lo que se tendría que esperar que a mayor número de bancos disminuyera el grado de concentración tanto en captación de depósitos como en otorgamiento de crédito, sin embargo, el sistema bancario sigue fuertemente concentrado.

El promedio del coeficiente de Gini para la concentración de depósitos es de 0.79 , esto indica una concentración más cercana al caso de monopolio. Para la concentración en el otorgamiento de crédito los resultados fueron muy similares a los obtenidos en el primer caso, el promedio del período es de $0.76 \%$ indicando también una situación más cercana al caso monopólico.

17 De acuerdo al padrón de banca múltiple registrado por la CNBV, en 2014 hay 43 bancos, que son los siguientes: ABC Capital, Actinver, Afirme, Agrofinanzas, American Express, Autofin, Banamex, Banca Mifel, Banco Ahorro Famsa, Banco Azteca, Banco Bancrea, Banco Base, Banco Bicentenario, Banco Credit Suisse, Banco del Bajío, Banco Wal-Mart, BanCoppel, Bank of America, Bank of Tokyo-Mitsubishi ufJ, Banorte, Banregio, Bansí, вBVA Bancomer, сіваnco, Compartamos, Consubanco, Deutsche Bank, Dondé Banco, Forjadores, HsBc, Inbursa, Inmobiliario Mexicano, Inter Banco, Interacciones, Invex, J.P. Morgan, Monex, Multiva, Santander, Scotiabank, The Royal Bank of Scotland, Ve por Más, Volkswagen Bank. 
Gráfica 2. Índice de Gini en la concentración de captación de depósitos y otorgamiento de crédito

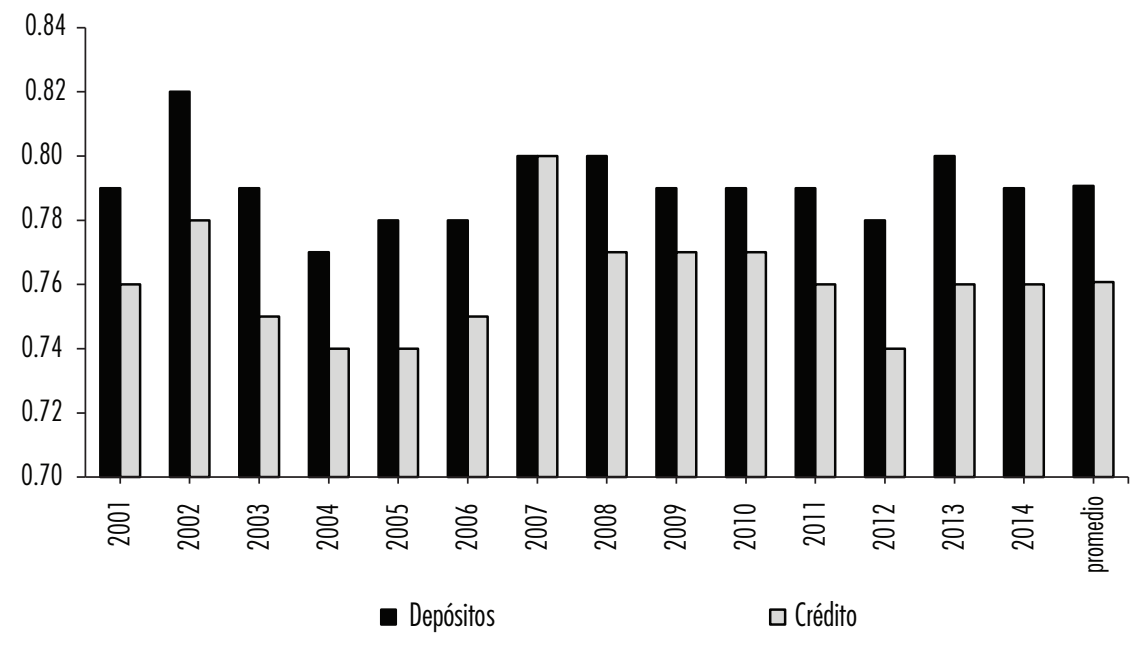

Fuente: elaborado con datos de la CNBV.

Si aceptamos la hipótesis de que la existencia de un mercado de crédito que no es competitivo, debilita los efectos multiplicadores de una política monetaria expansiva sobre el producto; entonces podemos decir que el sentido de la causalidad va de la concentración en el mercado de crédito hacia el crecimiento económico. Es decir, la relación entre estas dos variables debería ser inversa, a mayor índice de Gini menor crecimiento. En efecto, para el periodo 2001-2013, el coeficiente de correlación entre la tasa de crecimiento económico de México y la concentración en el otorgamiento de créditos es de $-0.28 \%$, mientras que, entre crecimiento económico y concentración de depósitos es de $-0.40 \% .^{18}$

El Banco de México señala en su "Reporte sobre las condiciones de competencia en los mercados financieros" (2013: 5) que: "un sector financiero competitivo coadyuva a que el financiamiento fluya a los proyectos más productivos en los mejores términos, que las personas realicen pagos en una forma eficiente y segura, y que sus ahorros obtengan los mejores rendimientos". ${ }^{19} \mathrm{Sin}$ embargo, con base en algunos indicadores de este reporte se puede deducir

18 Los coeficientes de correlación estimados se realizaron con base en datos del INEGI sobre la tasa de crecimiento anual del producto interno bruto, y los índices de Gini calculados en este trabajo.

19 Véase el "Reporte sobre las condiciones de competencia en los mercados financieros", 2 de mayo de 2013, p. 5, Banco de México. 
que existe un esquema de competencia imperfecta en el sistema bancario, por tanto el financiamiento no fluirá hacia los proyectos más productivos, habrá ineficiencia en los sistemas de pago y los ahorros no obtendrán los mejores rendimientos.

Para un sistema bancario competitivo, Bernanke (1983: 263) define el costo de intermediación del crédito (CCI) como el costo de canalizar fondos desde los ahorradores prestadores hasta las manos de los buenos prestatarios. El cCr incluye la proyección, monitoreo y los costos de contabilidad, así como las pérdidas esperadas ocasionadas por los malos prestatarios. Los bancos presumiblemente eligen procedimientos operativos que minimizan el CCI. Sin embargo, en un sistema bancario de competencia imperfecta como el que opera en México el costo de intermediación del crédito es muy alto, este lo podemos aproximar como la diferencia entre las tasas de interés activas (en este caso tomamos, para ejemplificar, la tasa de interés del crédito a los hogares) y pasivas (costo de captación promedio). El periodo que analizamos es de enero de 2005 a mayo de 2014, como se muestra en la gráfica 3 la brecha entre la tasa activa y pasiva es muy alta; de acuerdo con los datos del Banco de México, de 2005 a 2008 se ubicó en promedio en $9.66 \%$, para ascender a $10.76 \%$ de 2009 a 2014, ajuste que refleja la mayor percepción de riesgo asociada con la crisis financiera mundial de 2008-2009.

Gráfica 3. Tasas de interés activas y pasivas de la banca comercial en México

(Datos mensuales)

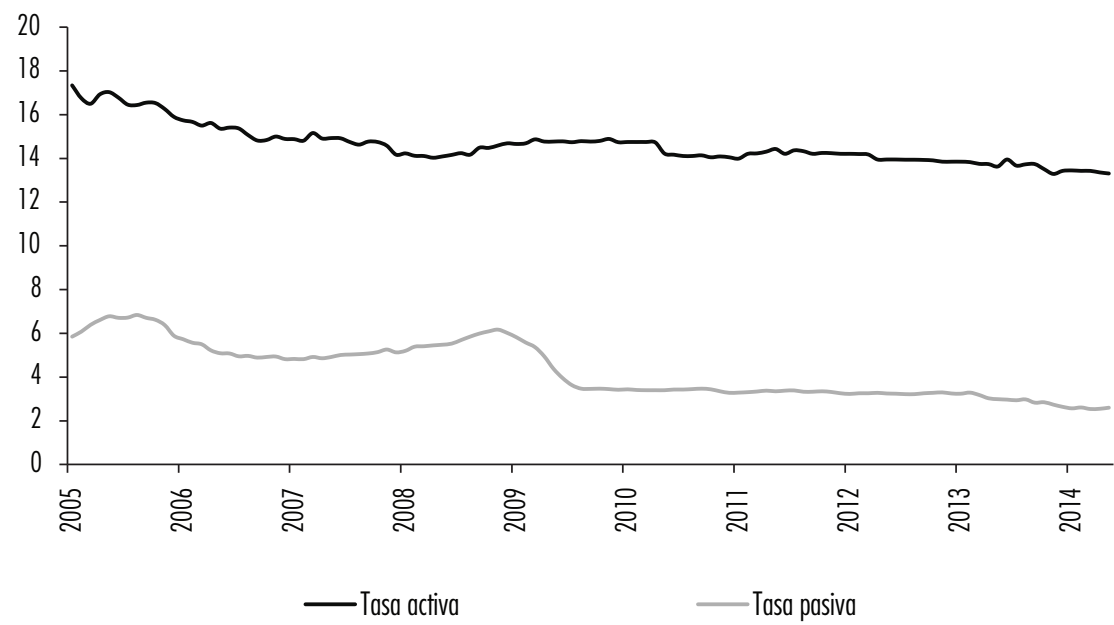

Fuente: elaborado con datos del Banco de México. 
Otro problema en México es el grave rezago en inclusión financiera, la CNBV define a la inclusión financiera como: “...el acceso y uso de servicios financieros bajo una regulación apropiada que garantice esquemas de protección al consumidor y promueva la educación financiera para mejorar las capacidades financieras de todos los segmentos de la población". Esta definición no sólo considera la posibilidad de acceso (oferta), sino el uso efectivo de los servicios financieros por parte de los individuos (demanda).

Peña, Hoyo y Tuesta (2014), analistas de Bвva Bancomer, señalan que de acuerdo a las estadísticas de los niveles de ahorro y la población que tiene una cuenta en una institución financiera en México, los niveles se ubican por debajo de América Latina y el Caribe y del promedio a nivel mundial. Durante 2011 en el país sólo el 27.4\% de la población mayor de 15 años tiene una cuenta en una institución financiera (un banco, unión de crédito o cooperativa), y un $7 \%$ realizó algún tipo de ahorro en alguna institución. Los datos anteriores son sólo una muestra de los muchos que los autores presentan para señalar el grave rezago en inclusión financiera. Su estudio se basa en datos de la Encuesta Nacional de Inclusión Financiera (ENIF) 2012, realizada por la CNBV y el Instituto Nacional de Estadística y Geografía (INEGI). Otros autores como Ampudia (2011) también muestran la exclusión financiera a la que está sujeta sobre todo la población más pobre y las micro, pequeñas y medianas empresas, debido al proceso de desbancarización y restricción crediticia.

Un problema más es el margen de ganancia que obtienen los bancos oligopólicos. Los datos del Banxico indican que, el costo de captación a plazo de pasivos en moneda nacional para abril de 2014 es de $3.41 \%$; mientras que el costo anual total promedio (СAT) sobre créditos hipotecarios durante marzo de 2014 fue de $13.43 \%$.

El destino de los créditos otorgados es un elemento que indica las preferencias de los bancos; parte del control que tienen sobre el mercado se refleja en la capacidad de decisión sobre el otorgamiento de crédito sólo a ciertas industrias. De 2001 a 2013 el monto del crédito de la banca comercial al sector privado del país es de 1,321,151.2 millones de pesos, de los cuales sólo el $2.26 \%$ fue destinado al sector agrícola (el cual se ve severamente castigado), mientras que el sector servicios recibió $27.0 \%$ del crédito total, como se muestra en la gráfica 4. 
Gráfica 4. Participación promedio en el otorgamiento de crédito de la banca comercial al sector privado de México (2001-2013)

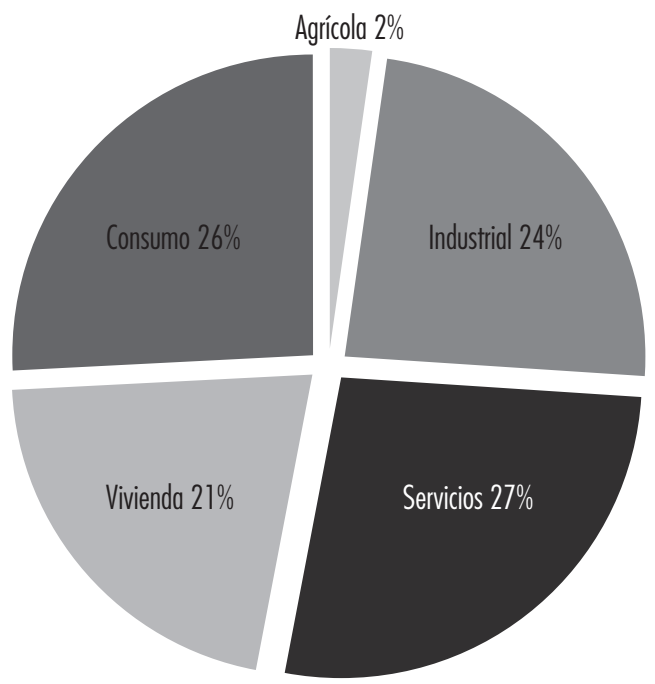

Fuente: elaborado con datos de Banxico.

\section{CONCLUSIONES}

En el marco de la teoría económica diferentes autores señalan que la generación de crédito en la economía es un factor que influye en el crecimiento económico, por esta razón dicha actividad no se debe dejar en manos exclusivamente del sector privado, de ahí que la banca de desarrollo debe de complementar las actividades de los bancos comerciales.

$\mathrm{Si}$ bien es cierto que existen varios factores que pueden explicar el lento crecimiento, el trabajo ha mostrado que en México el proceso de intermediación financiera a través del crédito bancario se ve limitado tanto por factores de oferta asociados a la existencia de un oligopolio bancario como por factores de demanda relacionados con la exclusión financiera.

Para alcanzar mayores tasas de crecimiento es necesario llevar a cabo un conjunto de políticas. Por ejemplo: eliminar el alto grado de informalidad en el sistema económico, esto generaría menores riesgos en el otorgamiento de créditos a las micro, pequeñas y medianas empresas; asimismo programas de apoyo para impulsar las exportaciones de este tipo de empresas. En opinión del director de los Fideicomisos Instituidos en Relación a la Agricultura (FIRA) se requiere una mayor competencia de los seguros agrícolas, más coberturas de precios flexibles que se puedan operar en cualquier momento de tal manera 
que haya proyectos más sólidos y que los intermediarios financieros estén dispuestos a correr el riesgo de otorgar crédito. ${ }^{20}$ Por tanto, considerando los elementos presentados en este trabajo, podemos afirmar que cualquier iniciativa que tenga como objetivo permitir mayor participación en el mercado de fondos prestables, deberá tener como columna vertebral la reducción del poder del oligopolio bancario.

Para otorgar un mayor número de créditos a menores tasas de interés es necesario hacer reformas estructurales sustanciales que realmente incidan en una mayor competencia en el mercado de crédito y de esta manera contribuir al bienestar social. Una alternativa es el fortalecimiento de la banca de desarrollo con la finalidad de impulsar el crecimiento de los sectores económico que no son preferentes para la banca comercial, como los pequeños agricultores y productores del sector primario, los cuales difícilmente pueden obtener un crédito en el sistema bancario y mucho menos en los mercados internacionales.

Es necesario señalar que en la reciente reforma financiera existe un capítulo dedicado a la banca de desarrollo, que reconoce que "la fortaleza de la banca de desarrollo permite detonar el crédito en actividades productivas y estratégicas que de otra manera no lo recibirían"; ${ }^{21}$ de igual forma se afirma que "los recursos del gobierno federal no son suficientes para financiar el desarrollo total del país, por ello la necesidad de potenciar los mismos y utilizarlos como inductores de la participación del sector privado, de forma eficiente". ${ }^{22}$ Sin embargo, desde la perspectiva de Juan Castaingts, la reforma financiera dejó en lo sustancial la fuerza del oligopolio bancario, no cambió en el fondo su obtención de ganancias, siguen buscando más el cobro de servicios que realizar su trabajo de recibir depósitos y dar créditos, las tasas de interés activas, las que realmente paga el acreedor, continúan siendo mucho más altas que las que recibe el ahorrador y además, son más elevadas en México que en el extranjero, lo cual afecta principalmente a las pequeñas y medianas empresas, las cuales son las mayores generadoras de empleos. ${ }^{23}$

El hecho de que la reforma financiera proponga el fortalecimiento de la banca de desarrollo como mecanismo para impulsar actividades estratégicas

20 Chávez, Héctor (2014), "Banca de desarrollo tiene que colaborar con instituciones privadas", El Financiero, p. 19, 4 de noviembre.

21 Véase Iniciativa de decreto por el que se reforman, adicionan y derogan diversas disposiciones de la Ley de Instituciones de Crédito, 8 de mayo de 2013.

22 Idem.

23 Véase “Reformas, ¿por qué no impulsan el crecimiento?”, El Financiero, 10 de abril, 2014. 
y deje de lado el poder oligopólico de la banca comercial, termina por ser una reforma incompleta. Toda política económica debe tener como objetivo último dirigir a la economía hacia una mejor situación social de la que prevalece, en este sentido el trabajo subraya el papel que los bancos comerciales desempeñan en el mecanismo de transmisión de la política monetaria y la capacidad que tienen para menguar o multiplicar los efectos de la política sobre las variables reales.

\section{BIBLIOGRAFÍA}

Ampudia Márquez, Nora (2011), "Exclusión financiera y desarrollo”, en Noemi Levy Orlik y Teresa López González (coords.), Las instituciones financieras y el crecimiento económico en el contexto de la dominación del capital financiero, México, Facultad de Economía unam y Juan Pablos Editor, pp. 159-180.

Bain, Keith y Peter Howells (2003), Monetary Economics: Policy and its Theoretical Basis, Gran Bretaña, Palgrave Macmillan.

Banco de México (2009), "Encuesta de evaluación coyuntural del mercado crediticio", disponible en www.banxico.com

(2013), "Reporte sobre las condiciones de competencia en los mercados financieros", disponible en http://www.banxico.org.mx/publicacionesdiscursos/index.html

Bernanke, Ben (1983), "Non-Monetary Effects of the Financial Crisis in the Propagation of the Great Depression", American Economic Review,73 (3), Estados Unidos, American Economic Association, pp. 257-273.

(2014), Mis años en la Reserva Federal. Un análisis de la Fed y las crisis financieras, México, Ediciones Deusto.

y Alan Blinder (1988), "Credit, Money and Aggregate Demand", American Economic Review, Paper and Proceedings, 78 (2), EstadosUnidos, American Economic Association, pp. 435-439.

y Mark Gertler (1995), "Inside the Black Box: The Credit Channel of Monetary Policy Transmission", Journal of Economic Perspectives 9 (4), Estados Unidos, American Economic Association, pp. 27-48.

Brunner, Karl y Allan Meltzer (1995), El dinero y la economía, España, Alianza Editorial.

De Gregorio Rebeco, José (2007), Macroeconomía, teoría y politica, México, Pearson Educación. 
Fisher, Irving (1973), "I Discovered the Phillips Curve: A Statistical Relation between Unemployment and Price Changes", Journal of Political Economy, vol. 81, núm. 2, Part 1 Estados Unidos, March-April, pp. 496-502.

(1928), The Money Illusion, Nueva York, Estados Unidos, Adelphi Company.

Friedman, Milton (1976), "Inflación y desempleo", en Los premios Nobel de Economía, 1969-1977, México, El Trimestre Económico, Fondo de Cultura Económica (25), pp. 313-340.

(1968), "The Role of Monetary Policy", American Economic Review, 58 (1), Estados Unidos, American Economic Association, March, pp. 1-17. y Anna Schwartz (1963), A Monetary History of the United States, 1867-1960, Estados Unidos, Princeton University Press.

Hawtrey, R. G. (1928), Trade and Credit, Londres, Toronto, Longmans, Green.

Holt, R.P.F. (1997), "Post Keynesian School of Economics", en T. Cate (coord.), An Encyclopedia of Keynesian Economics, Cheltenham, UK y Lyme, USA, Edward Elgar.

Keynes, John M. (1996[1930]), Tratado del dinero, España, Biblioteca de Grandes Economistas del siglo xx, Ediciones Aosta.

(1984 [1936]), Teoría general de la ocupación, el interés y el dinero, México, Fondo de Cultura Económica.

(1937a), "Alternative Theories of the Rate of Interest", The Economic Journal, vol. 47, núm. 186, Estados Unidos, junio, pp. 241-252.

(1937b), "The Ex-ante Theory of the Rate of Interest", The Economic Journal, vol. 47, núm. 188, Estados Unidos, diciembre, pp. 663-692.

(1939), "The Process of Capital Formation", en The Economic Journal, reimpreso en J. M. Keynes, Collected Writings, Londres, D.E. Moggridge: Macmillan, for The Royal Economic Society, pp. 278-285.

Larraín, Felipe y Jeffrey Sachs (2013), Macroeconomía en la economía global, Tercera edición, Chile, Pearson Education.

Lavoie, Marc (2014), La economía poskeynesiana, Barcelona, España, Editorial Icaria Antrazyt.

León León, Josefina (2012), "Reflexiones críticas sobre el mecanismo de transmisión de la política monetaria del Banco de México", en Alma Chapoy y Patricia Rodríguez (coords.), Tras la crisis, politicas públicas a favor del crecimiento económico, México, UnAM, pp. 31-50.

(2010), "Aportaciones al pensamiento monetario actual: tasa de interés de R.G. Hawtrey y su influencia en J.M. Keynes”, en Alicia Girón, 
Eugenia Correa y Patricia Rodríguez (coords.), Pensamiento poskeynesiano, de la inestabilidad financiera a la reestructuración macroeconómica", México, UNAM, IIEc, pp. 95-134.

Levy Orlik, Noemi (2011), "Las estructuras financieras y el financiamiento de la producción en los principales países latinoamericanos", en Noemi Levy Orlik y Teresa López González (coords.), Las instituciones financieras y el crecimiento económico en el contexto de la dominación del capital financiero, México, Facultad de Economía unam, y Juan Pablos Editor, pp. 111-148. Mishkin, Frederic (1995), "Symposium on the Monetary Transmission Mechanism", Journal of Economic Perspective, Estados Unidos 9 (9), pp. 3-10.

Nasar, Sylvia (2014), La gran buisqueda. Historia de los genios económicos que cambiaron el mundo, México, DEBATE.

Peña, Ximena, Carmen Hoyo y David Tuesta (2014), "Determinantes de la inclusión financiera en México a partir de la Encuesta Nacional de Inclusión Financiera (ENIF) 2012”, BBVA Research, Documento de Trabajo, No. 14/14, Madrid.

Perrotini Hernández, Ignacio (2007), "El nuevo paradigma monetario", en Economíanam, 4 (11), México, Facultad de Economía, unam, mayoagosto, pp. 64-82.

Piégay, Pierre y Louis Philippe Rochón (coords.) (2006), Teorias monetarias poskeynesianos, España, Ediciones Akal.

Secretaría de Hacienda y Crédito Público (2013), "Iniciativa de Decreto por el que se reforman, adicionan y derogan diversas disposiciones de la Ley de Instituciones de Crédito", disponible en http://www.shcp.gob.mx/Paginas/default.aspx

Snowdon, Brian y Howard R. Vane (2005), Modern Macroeconomics, Estados Unidos, Edward Elgar.

Vega, Juan Luis (1992), "El papel del crédito en el mecanismo de transmisión monetaria”, Documento de trabajo núm. 48, Banco de España, España, disponible en www.bde.es/informes/be/sazul/azul48.pdf 
